\title{
Energy Flux Correlations and Moving Mirrors
}

\author{
L.H. Ford* \\ Institute of Cosmology \\ Department of Physics and Astronomy \\ Tufts University, Medford, MA 02155 \\ Thomas A. Romant \\ Department of Mathematical Sciences \\ Central Connecticut State University \\ New Britain, CT 06050
}

\begin{abstract}
We study the quantum stress tensor correlation function for a massless scalar field in a flat two-dimensional spacetime containing a moving mirror. We construct the correlation functions for right-moving and left-moving fluxes for an arbitrary trajectory, and then specialize them to the case of a mirror trajectory for which the expectation value of the stress tensor describes a pair of delta-function pulses, one of negative energy and one of positive energy. The flux correlation function describes the fluctuations around this mean stress tensor, and reveals subtle changes in the correlations between regions where the mean flux vanishes.
\end{abstract}

PACS numbers: 03.65.Ud, 04.62.+v, 03.70.+k

*Email: ford@cosmos.phy.tufts.edu

†Email: roman@ccsu.edu 


\section{INTRODUCTION}

In recent years, there has been a considerable amount of interest in quantum violations of the weak energy condition and the inequalities which constrain such violations [1, 2, 3, 4, 4, 6, 6, 7, 8, 9]. The Fulling-Davies model [10, 11] of a moving mirror in two-dimensional spacetime provides a useful model for the study of such violations. In particular, when the mirror is moving with increasing acceleration to the right, a flux of negative energy is emitted to the right. Similarly, when the acceleration to the right is decreasing, a positive energy flux is emitted in this direction. This model provides a simple illustration of how negative energy fluxes obey quantum inequalities, and of the phenomenon of quantum interest [12]. The Fulling-Davies model has been used by several authors to study the phenomenon of black hole evaporation, as there exists a specific trajectory which produces the same outgoing quantum state as does an evaporating black hole in two-dimensions. Several authors [13, 14, 15, 16] have used the mirror model to study correlations between particles emitted in opposite directions, which in the black hole case are the correlations between particles emitted to infinity and those absorbed by the black hole.

In this paper, our interest will be the correlations between the energy fluxes moving along different null lines, rather than correlations between particles. The positive and negative energy fluxes emitted by a moving mirror are really mean fluxes given by the expectation value of the quantum stress tensor. However, the quantum state is not an eigenstate of the stress tensor operator, so there should be fluctuations around this mean value. Quantum stress tensor fluctuations and their role in gravity theory are still not well understood, but are an active area of investigation [17, 18, 19, 20, 21, 22, 23, 24, 25, 26, 27]. Even in the Minkowski vacuum state, there will be stress tensor fluctuations with subtle correlations. The expectation value of the stress tensor vanishes everywhere, yet an individual measurement in a finite region can return a negative value. Somehow these fluctuations must be constrained so as not to produce dramatic observable effects. Similarly, one expects that even in situations where the expectation value of the stress tensor violates the weak energy condition, there will be restrictions upon how much additional negative energy can be observed in a given fluctuation. However, this is not yet well understood.

In general, the study of the effects of quantum stress tensor fluctuations requires one to deal with the singular short distance behavior of the stress tensor correlation function. One approach is to discuss only spacetime averages. It is possible by a careful treatment to give a meaning to the formally divergent integrals of the correlation function. An alternative approach is to restrict our attention to the correlation between stress tensors in disjoint regions of spacetime, which will be adopted in the present paper. We will examine a particular mirror trajectory in detail. This is a mirror which is initially static, abruptly begins to accelerate, emitting a delta-function pulse of negative mean energy. The mirror subsequently stops accelerating, emitting a larger delta-function pulse of positive mean energy, and henceforth moves at constant velocity. The expectation value of the stress tensor operator is nonzero only along the outgoing null rays emanating from the points where the acceleration changes abruptly, and is zero elsewhere, including during the constant acceleration phase. Our aim is to understand the correlations between fluctuations in the flux, including in regions where the mean flux vanishes. Obadia and Parentani [16] have previously examined the correlations between incoming and outgoing flux fluctuations for an eternally accelerating mirror, where the expectation value of the stress tensor vanishes everywhere.

One question of interest is the degree of correlation or anticorrelation of the negative and positive pulses. The time-integrated energy seen by any inertial observer must be nonnegative in any individual measurement [28]. This follows from the fact that the integrated flux is described by an operator whose spectrum is bounded below by zero. Thus not only must the integral of the expectation value of the flux be non-negative, but in each measure- 
ment the observer must detect at least as much positive as negative energy. Now consider the situation where a fluctuation makes the negative pulse considerably more negative than its mean value. There must be a corresponding upward fluctuation in the flux somewhere else to guarantee a net positive integrated energy. Although we partially answer this question in the present paper, our main focus will be to show that there are nontrivial changes in the correlations across pulses.

The outline of the paper is as follows: In Sect III we construct the various correlation functions for the right and left-moving fluxes in the presence of a mirror undergoing arbitrary motion. In Sect. III, the explicit forms of the correlation functions are constructed for our specific mirror trajectory. Various limits of these functions are studied and interpreted. Our results are summarized and discussed in Sect. IV

\section{CORRELATION FUNCTIONS FOR A GENERAL MIRROR TRAJECTORY}

In this paper, we will consider a massless scalar field in two-dimensional spacetime, for which the stress tensor operator is

$$
T_{\mu \nu}=\varphi_{, \mu} \varphi_{, \nu}-\frac{1}{2} g_{\mu \nu} \varphi_{, \rho} \varphi^{, \rho} .
$$

The energy flux to the right is given by

$$
T_{u u}=\varphi_{, u} \varphi_{, u},
$$

and that to the left is

$$
T_{v v}=\varphi_{, v} \varphi_{, v},
$$

where $u=t-x$ and $v=t+x$ are null coordinates.

We now wish to construct the stress tensor correlation functions for this theory. First consider the correlation between two right-moving rays

$$
C_{R R}\left(u, u^{\prime}\right)=\left\langle T_{u u}(u) T_{u u}\left(u^{\prime}\right)\right\rangle-\left\langle T_{u u}(u)\right\rangle\left\langle T_{u u}\left(u^{\prime}\right)\right\rangle .
$$

Here the quantum state will be taken to be the in-vacuum state, the state with no particles

at early times. For this, or any other choice of vacuum state, one may show using Wick's theorem that

$$
\left\langle\varphi_{1} \varphi_{2} \varphi_{3} \varphi_{4}\right\rangle=\left\langle\varphi_{1} \varphi_{2}\right\rangle\left\langle\varphi_{3} \varphi_{4}\right\rangle+\left\langle\varphi_{1} \varphi_{3}\right\rangle\left\langle\varphi_{2} \varphi_{4}\right\rangle+\left\langle\varphi_{1} \varphi_{4}\right\rangle\left\langle\varphi_{2} \varphi_{3}\right\rangle
$$

where $\varphi_{1}$, etc are field operators or their derivatives. Let $\varphi_{1}=\varphi_{2}=\partial_{u} \varphi(u)$ and $\varphi_{3}=\varphi_{4}=$ $\partial_{u^{\prime}} \varphi\left(u^{\prime}\right)$. Then we have

$$
C_{R R}\left(u, u^{\prime}\right)=2\left[\partial_{u} \partial_{u^{\prime}} D\left(y, y^{\prime}\right)\right]^{2}
$$

where

$$
D\left(y, y^{\prime}\right)=\left\langle\varphi(y), \varphi\left(y^{\prime}\right)\right\rangle
$$

is the two-point function for the quantized scalar field for the spacetime points $y=(u, v)$ and $y^{\prime}=\left(u^{\prime}, v^{\prime}\right)$.

Similarly, we may construct the correlation function between two left-moving rays as

$$
C_{L L}\left(v, v^{\prime}\right)=\left\langle T_{v v}(v) T_{v v}\left(v^{\prime}\right)\right\rangle-\left\langle T_{v v}(v)\right\rangle\left\langle T_{v v}\left(v^{\prime}\right)\right\rangle
$$


and that between a right moving ray and a left-moving ray as

$$
C_{R L}\left(v, u^{\prime}\right)=\left\langle T_{v v}(v) T_{u u}\left(u^{\prime}\right)\right\rangle-\left\langle T_{v v}(v)\right\rangle\left\langle T_{u u}\left(u^{\prime}\right)\right\rangle .
$$

These functions may be expressed in terms of the two-point function as

$$
C_{L L}\left(v, v^{\prime}\right)=2\left[\partial_{v} \partial_{v^{\prime}} D\left(y, y^{\prime}\right)\right]^{2},
$$

and

$$
C_{R L}\left(v, u^{\prime}\right)=2\left[\partial_{u^{\prime}} \partial_{v} D\left(y, y^{\prime}\right)\right]^{2}
$$

The two-point function in the region to the right of a moving mirror is given by [29]

$$
D=-\frac{1}{4 \pi} \ln \left\{\frac{\left[p(u)-p\left(u^{\prime}\right)-i \epsilon\right]\left(v-v^{\prime}-i \epsilon\right)}{\left[v-p\left(u^{\prime}\right)-i \epsilon\right]\left[p(u)-v^{\prime}-i \epsilon\right]}\right\} .
$$

Here the function $p(u)$ describes the reflection of null rays by the mirror: an incoming $v=$ constant ray reflects from the mirror and becomes an outgoing $u=$ constant ray, where the values of $u$ and $v$ are related by $v=p(u)$, as illustrated in Fig. 1] Because we deal only with the correlations of distinct rays, for which $u \neq u^{\prime}$ and $v \neq v^{\prime}$, we can drop the $i \epsilon$ terms in the above expression. Some explicit forms for $p(u)$ will be given in the next section. We can now show that

$$
\begin{aligned}
C_{R R}\left(u, u^{\prime}\right) & =\frac{\left[p^{\prime}\left(u^{\prime}\right) p^{\prime}(u)\right]^{2}}{8 \pi^{2}\left[p\left(u^{\prime}\right)-p(u)\right]^{4}}, \\
C_{R L}\left(v, u^{\prime}\right) & =\frac{\left[p^{\prime}\left(u^{\prime}\right)\right]^{2}}{8 \pi^{2}\left[p\left(u^{\prime}\right)-v\right]^{4}},
\end{aligned}
$$

and

$$
C_{L L}\left(v, v^{\prime}\right)=\frac{1}{8 \pi^{2}\left(v^{\prime}-v\right)^{4}}
$$

where $p^{\prime}(u)=d p / d u$, etc.

Note that the correlation function $C_{L L}\left(v, v^{\prime}\right)$ is independent of the details of the motion of the mirror, that is, of the form of the function $p(u)$. This is to be expected. The correlations between the incoming fluxes should not depend upon whether a mirror is present or not, and if so, whether it is moving. Thus the above form for $C_{L L}\left(v, v^{\prime}\right)$ is the same as that for a static mirror or for empty Minkowski spacetime without a mirror. In the limit of a static mirror, $v=p(u)=u$, and

$$
C_{R R}\left(u, u^{\prime}\right)=C_{v a c}\left(u, u^{\prime}\right)=\frac{1}{8 \pi^{2}\left(u^{\prime}-u\right)^{4}}
$$

and

$$
C_{R L}\left(v, u^{\prime}\right)=C_{\text {static }}\left(v, u^{\prime}\right)=\frac{1}{8 \pi^{2}\left(u^{\prime}-v\right)^{4}} .
$$

The above limit of $C_{R R}\left(u, u^{\prime}\right)$ is the same for a static mirror and for empty Minkowski spacetime, but the limit for $C_{R L}\left(v, u^{\prime}\right)$ applies only to a static mirror. In empty Minkowski spacetime, $C_{R L}\left(v, u^{\prime}\right)=0$, as there can only be correlations between right and left moving fluxes if there is a mirror present.

We can understand the general structure of $C_{R L}\left(v, u^{\prime}\right)$ and $C_{R R}\left(u, u^{\prime}\right)$ as follows. To go from Eq. (15) for $C_{L L}\left(v, v^{\prime}\right)$ to Eq. (14) for $C_{R L}\left(v, u^{\prime}\right)$, we replace $v^{\prime} \rightarrow p\left(u^{\prime}\right)$ and multiply by $\left[p^{\prime}\left(u^{\prime}\right)\right]^{2}$. The $v^{\prime} \rightarrow p\left(u^{\prime}\right)$ replacement simply describes the mapping of the left-moving to a right-moving ray as it reflects from the mirror. The $\left[p^{\prime}\left(u^{\prime}\right)\right]^{2}$ factor is a Doppler shift factor, which describes the redshift or blueshift of an energy flux as it reflects from a moving mirror. Similarly, we can go from Eq. (15) for $C_{L L}\left(v, v^{\prime}\right)$ to Eq. (13) for $C_{R R}\left(u, u^{\prime}\right)$ by the double replacement $v \rightarrow p(u)$ and $v^{\prime} \rightarrow p\left(u^{\prime}\right)$, corresponding to the reflection of two left-moving rays, and multiplication by the Doppler shift factors $\left[p^{\prime}(u)\right]^{2}$ and $\left[p^{\prime}\left(u^{\prime}\right)\right]^{2}$. 


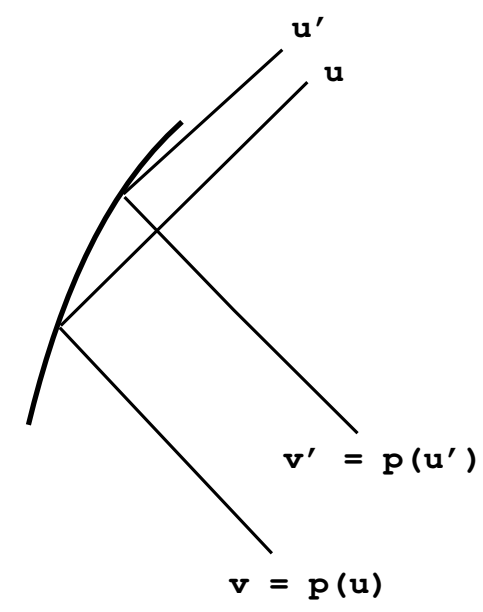

FIG. 1: The reflection of null rays by a moving mirror is illustrated. A left-moving ray with $v=$ constant reflects from the mirror and becomes a right-moving ray with $u=$ constant, where $v=p(u)$. Another left-moving ray $v^{\prime}$ becomes a right-moving ray $u^{\prime}$ with $v^{\prime}=p\left(u^{\prime}\right)$. Note that if the mirror is moving to the right, $\Delta v=v^{\prime}-v>\Delta u=u^{\prime}-u$.

\section{A TRAJECTORY WITH PIECEWISE LINEAR ACCELERATION}

\section{A. Construction of $p(u)$}

As first shown by Fulling and Davies [10, 11], the expectation value of the $u$-component of the stress tensor to the right of the mirror is given by

$$
\left\langle T_{u u}\right\rangle=\frac{1}{12 \pi}\left(p^{\prime}\right)^{1 / 2} \partial_{u}^{2}\left(p^{\prime}\right)^{-1 / 2}=\frac{1}{24 \pi}\left[\frac{3}{2}\left(\frac{p^{\prime \prime}}{p^{\prime}}\right)^{2}-\frac{p^{\prime \prime \prime}}{p^{\prime}}\right] .
$$

We know that $\left\langle T_{u u}\right\rangle=0$ when the mirror moves either inertially or with constant acceleration. The trajectory we will construct corresponds to an initially static mirror at $x=0$, which begins to accelerate to the right at time $t=0$. The mirror then moves with constant acceleration until some later time when it stops accelerating and moves with constant velocity. The mirror will: (1) emit a $\delta$-function pulse of $(-)$ energy when it starts to accelerate at $t=0,(2)$ emit no radiation during the constant acceleration phase of the trajectory, and (3) emit a $\delta$-function pulse of $(+)$ energy when it stops accelerating. This trajectory is depicted in Fig. 2 .

Let us rewrite Eq. (18) in terms of the variable $q=p^{\prime}$, and solve the resulting equation when we set $\left\langle T_{u u}\right\rangle=0$. We have

$$
q q^{\prime \prime}-\frac{3}{2}\left(q^{\prime}\right)^{2}=0
$$

If we let

$$
q(u)=\frac{1}{\left(c_{1} u+c_{0}\right)^{2}},
$$






FIG. 2: A mirror starts from rest at $t=0$ and then abruptly begins to accelerate to the right with constant acceleration. At this time, the mirror emits a delta-function pulse of negative energy along the line $u=0$. At a later time, the mirror abruptly stops accelerating and emits a delta-function pulse of positive energy along the line $u=u_{f}$. Subsequently, the mirror moves with a constant velocity. Region 1 is $u<0$, before the acceleration starts. Region 2 is $0<u<u_{f}$, while the acceleration is constant, but no mean energy is emitted. Region 3 is $u>u_{f}$, after the acceleration stops.

where $c_{1}, c_{0}$ are constants, then

$$
p(u)=\frac{1}{a_{1} u+a_{0}}+a_{2},
$$

where $a_{1}, a_{0}$ and $a_{2}$ are constants. This describes the most general, uniformly accelerating trajectory, for which $\left\langle T_{u u}\right\rangle=0$. In the special case when $c_{1} \rightarrow \infty$, we get the solution

$$
p(u)=a_{3} u+a_{4},
$$

where $a_{3}$ and $a_{4}$ are constants, which describes an inertial mirror.

Assume that

$$
p(u)=\left\{\begin{array}{cc}
u, & u \leq 0 \\
1 /\left(a_{1} u+a_{0}\right)+a_{2}, & 0 \leq u \leq u_{f} \\
a_{3} u+a_{4}, & u \geq u_{f}
\end{array}\right.
$$

where $u \leq 0$ and $u \geq u_{f}$ correspond to the initial and final inertial phases of the trajectory, and $0 \leq u \leq u_{f}$ represents the constant acceleration part of the trajectory. Continuity of $p$ and $p^{\prime}$ at $u=0$ and $u=u_{f}$ yields

$$
\begin{aligned}
& a_{1}=-a_{0}^{2}, \\
& a_{2}=-1 / a_{0}, \\
& a_{3}=1 /\left(1-a_{0} u_{f}\right)^{2}, \\
& a_{4}=-a_{0} u_{f}^{2} /\left(1-a_{0} u_{f}\right)^{2} .
\end{aligned}
$$


Therefore, Eq. (23) becomes

$$
p(u)=\left\{\begin{array}{cc}
u, & u \leq 0 \quad(\text { region 1) } \\
u /\left(1-a_{0} u\right), & 0 \leq u \leq u_{f} \text { (region 2) }, \\
\left(u-a_{0} u_{f}^{2}\right) /\left(1-a_{0} u_{f}\right)^{2}, & u \geq u_{f} \quad(\text { region 3) }
\end{array}\right.
$$

and we have that

$$
\begin{gathered}
p^{\prime}(u)=\left\{\begin{array}{cc}
1, & u \leq 0 \\
1 /\left(1-a_{0} u\right)^{2}, & 0 \leq u \leq u_{f} \\
1 /\left(1-a_{0} u_{f}\right)^{2}, & u \geq u_{f}
\end{array}\right. \\
p^{\prime \prime}(u)=\left\{\begin{array}{cc}
0, & u<0 \\
2 a_{0} /\left(1-a_{0} u\right)^{3}, & 0<u<u_{f} \\
0, & u>u_{f}
\end{array}\right.
\end{gathered}
$$

and

$$
p^{\prime \prime \prime}(u)=p_{D}^{\prime \prime \prime}(u)+2 a_{0} \delta(u)-2 a_{0} \delta\left(u-u_{f}\right) /\left(1-a_{0} u_{f}\right)^{3},
$$

with

$$
p_{D}^{\prime \prime \prime}(u)=\left\{\begin{array}{cc}
0, & u<0 \\
6 a_{0}^{2} /\left(1-a_{0} u\right)^{4}, & 0<u<u_{f} \\
0, & u>u_{f}
\end{array} .\right.
$$

Substitution of our expressions for $p^{\prime}, p^{\prime \prime}$, and $p^{\prime \prime \prime}$ into Eq. (18) gives

$$
\left\langle T_{u u}\right\rangle=\frac{1}{12 \pi}\left[-a_{0} \delta(u)+\frac{a_{0}}{1-a_{0} u_{f}} \delta\left(u-u_{f}\right)\right] .
$$

The constant $a_{0}$ is the proper acceleration. The case $a_{0}>0$ corresponds to the acceleration of the mirror to the right, with an initial $(-)$ energy $\delta$-function pulse emitted to the right followed by a similar $(+)$ pulse to the right. Since the velocity of the mirror must always be less than the speed of light, we must require that $u_{f}<1 / a_{0}$. For $a_{0}<0$, the mirror accelerates to the left, which corresponds to an initial $(+)$ energy pulse to the right followed by a (-) energy pulse to the right. In this case, there is no restriction on $u_{f}$. The factor $1 /\left(1-a_{0} u_{f}\right)$ is a Doppler shift factor which is greater than 1 for $a_{0}>0$ and smaller than 1 for $a_{0}<0$. This factor guarantees than an initial (-) energy pulse must always be followed by a more than compensating $(+)$ energy pulse, an example of the phenomenon of quantum interest [12].

\section{B. Construction of the $C_{R R}$ 's}

Here we will temporarily drop the $R R$ subscripts in favor of numerical subscripts which indicate the region of location of each ray in Fig. 2. For example, $C_{12}\left(u, u^{\prime}\right)$ is the $R R$ correlation function with $u$ in region 1 and $u^{\prime}$ in region 2. In the case of the moving mirror, if we substitute Eqs. (28) and (29) into Eq. (13), we obtain the following expressions:

$$
C_{11}\left(u, u^{\prime}\right)=C_{22}\left(u, u^{\prime}\right)=C_{33}\left(u, u^{\prime}\right)=\frac{1}{8 \pi^{2}\left(u^{\prime}-u\right)^{4}},
$$


and

$$
\begin{aligned}
C_{12}\left(u, u^{\prime}\right) & =\frac{1}{8 \pi^{2}\left(u-u^{\prime}-a_{0} u u^{\prime}\right)^{4}} \\
C_{23}\left(u, u^{\prime}\right) & =\frac{\left(1-a_{0} u_{f}\right)^{4}}{8 \pi^{2}\left[u\left(1-a_{0} u_{f}\right)^{2}-\left(1-a_{0} u\right)\left(u^{\prime}-a_{0} u_{f}^{2}\right)\right]^{4}} \\
C_{13}\left(u, u^{\prime}\right) & =\frac{\left(1-a_{0} u_{f}\right)^{4}}{8 \pi^{2}\left[u\left(1-a_{0} u_{f}\right)^{2}-u^{\prime}+a_{0} u_{f}^{2}\right]^{4}} .
\end{aligned}
$$

Notice that in all cases when $u, u^{\prime}$ lie in the same region, the correlation function, $C_{R R}$, has the same form as in the vacuum. This includes the case $C_{22}$, where both rays are in the region of uniform acceleration. We show in the Appendix that uniform acceleration is the most general trajectory for which $C_{R R}\left(u, u^{\prime}\right)=C_{v a c}\left(u, u^{\prime}\right)$. This of course includes an inertial trajectory as a special case.

\section{Behavior of the $C_{R R}$ 's in different regions}

We saw in Sect. IIIB that in all cases when $u, u^{\prime}$ lie in the same region, the correlation function, $C_{R R}$, has the same form as in the vacuum. This is a consequence of the fact that the most general case for which $C_{R R}\left(u, u^{\prime}\right)=C_{v a c}\left(u, u^{\prime}\right)$ is constant acceleration, with constant velocity as a special subcase. Referring to Fig. 2. when rays $u, u^{\prime}$ are both in the constant velocity regions 1 or 3 , the mirror has zero acceleration. When both rays are in region 2 , the mirror is undergoing constant acceleration. Only pairs of outgoing rays which are on opposite sides of one or both energy pulses can have $C_{R R}\left(u, u^{\prime}\right) \neq C_{v a c}\left(u, u^{\prime}\right)$.

Let us now look at $C_{R R} / C_{v a c}$ for the remaining cases, where

$$
\begin{aligned}
& \frac{C_{12}\left(u, u^{\prime}\right)}{C_{v a c}}=\frac{\left(u-u^{\prime}\right)^{4}}{\left(u-u^{\prime}-a_{0} u u^{\prime}\right)^{4}}, \\
& \frac{C_{23}\left(u, u^{\prime}\right)}{C_{v a c}}=\frac{\left(1-a_{0} u_{f}\right)^{4}\left(u-u^{\prime}\right)^{4}}{\left[u\left(1-a_{0} u_{f}\right)^{2}-\left(1-a_{0} u\right)\left(u^{\prime}-a_{0} u_{f}^{2}\right)\right]^{4}}, \\
& \frac{C_{13}\left(u, u^{\prime}\right)}{C_{v a c}}=\frac{\left(1-a_{0} u_{f}\right)^{4}\left(u-u^{\prime}\right)^{4}}{\left[u\left(1-a_{0} u_{f}\right)^{2}-u^{\prime}+a_{0} u_{f}^{2}\right]^{4}} .
\end{aligned}
$$

We will consider primarily the case $a_{0}>0$, i. e., an initial $(-)$ energy pulse followed by a $(+)$ energy pulse. The case $a_{0}<0$ will be discussed briefly at the end of this subsection. Thus we see from Eq. (38) that $C_{12}\left(u, u^{\prime}\right) / C_{v a c}>1$. One can interpret this as saying that the $(-)$ energy pulse enhances the correlations of a pair of outgoing rays which lie on opposite sides of the pulse.

Now consider $C_{23}\left(u, u^{\prime}\right) / C_{v a c}$. For $u^{\prime}=u_{f}$, this function is equal to 1 ; it then falls monotonically as a function of $u^{\prime}$, for $u$ fixed and $u^{\prime}>u_{f}$. To verify the latter property, proceed as follows. Let

$$
\begin{aligned}
f\left(u^{\prime}\right) & =\left[\frac{C_{23}\left(u, u^{\prime}\right)}{C_{v a c}}\right]^{1 / 4} \\
& =\frac{\left(1-a_{0} u_{f}\right)\left(u^{\prime}-u\right)}{\left[\left(1-a_{0} u\right)\left(u^{\prime}-a_{0} u_{f}^{2}\right)-u\left(1-a_{0} u_{f}\right)^{2}\right]} .
\end{aligned}
$$




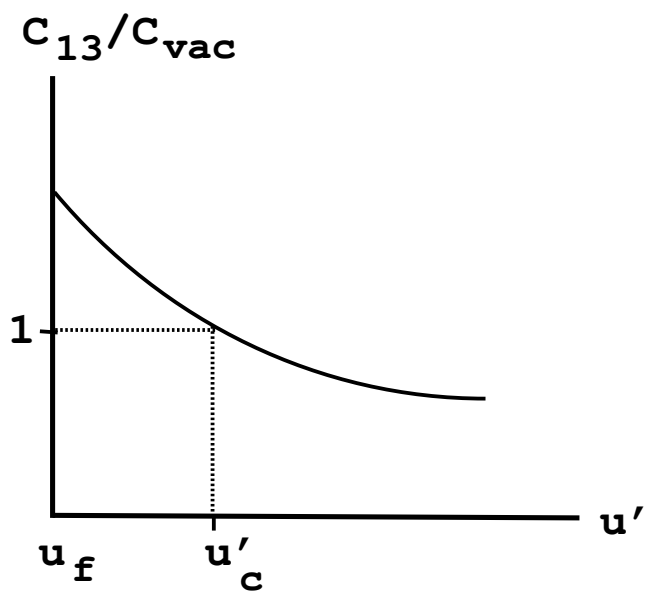

FIG. 3: The behavior of the ratio $C_{13}\left(u, u^{\prime}\right) / C_{v a c}\left(u, u^{\prime}\right)$ as a function of $u^{\prime}$ for fixed $u$ is illustrated for $a_{0}>0$. Here $u<0$ and $u^{\prime} \geq u_{f}$. The ratio starts at a value greater than one at $u^{\prime}=u_{f}$ and then falls monotonically, passing through one at $u^{\prime}=u_{c}^{\prime}$.

We first check that the denominator in Eq. (42) is always positive. Since $\left(1-a_{0} u\right)>0$ (recall that $\left.u<1 / a_{0}\right)$, the denominator is a monotone increasing function of $u^{\prime}$, for fixed $u$. Furthermore, at $u^{\prime}=u_{f}$ this function is equal to $\left(1-a_{0} u_{f}\right)\left(u_{f}-u\right)>0$, which is consistent with $f\left(u^{\prime}\right)>0$. This confirms that we took the correct sign for the fourth root in Eq. (42). The derivative of $f\left(u^{\prime}\right)$ is

$$
f^{\prime}\left(u^{\prime}\right)=-a_{0} \frac{\left(1-a_{0} u_{f}\right)\left(u_{f}-u\right)^{2}}{\left[\left(1-a_{0} u\right)\left(u^{\prime}-a_{0} u_{f}^{2}\right)-u\left(1-a_{0} u_{f}\right)^{2}\right]^{2}}<0 .
$$

However, $f\left(u_{f}\right)=1$ while $f^{\prime}\left(u^{\prime}\right)<0$, so $f\left(u^{\prime}\right)$ and hence $C_{23}\left(u, u^{\prime}\right) / C_{v a c}$, decreases monotonically from 1 as $u^{\prime}$ increases from $u_{f}$. The fact that $C_{23}\left(u, u^{\prime}\right) / C_{v a c}<1$ for $u^{\prime}>u_{f}$ indicates that a $(+)$ energy pulse suppresses correlations across the pulse.

An argument similar to that given for $C_{23}\left(u, u^{\prime}\right) / C_{v a c}$ shows that $C_{13}\left(u, u^{\prime}\right) / C_{v a c}$ is a monotonically decreasing function of $u^{\prime}$, for fixed $u$. This ratio starts at a value greater than 1, a fact which follows from continuity and the fact that $C_{12}\left(u, u_{f}\right) / C_{v a c}>1$. Eventually, $C_{13}\left(u, u^{\prime}=u_{c}^{\prime}\right) / C_{v a c}=1$, at $u_{c}^{\prime}=u_{f}-u\left(1-a_{0} u_{f}\right)$, as shown in Fig. 3. The fact that $C_{13}\left(u, u^{\prime}\right) / C_{v a c}$ eventually falls below 1 implies that the effect of both pulses together is to suppress correlations, provided that the second ray $u^{\prime}$ is not too close to the $(+)$ pulse. One can think of $u_{c}^{\prime}$ as the "time delay" before the effects of the $(+)$ pulse come to dominate those of the $(-)$ pulse. Note that since here $u<0, u_{c}^{\prime}$ grows as $u \rightarrow-\infty$. This behavior will be discussed in Sec. IIIE.

\section{Limits with large pulses}

There are two ways in which the magnitudes of the pulses can become arbitrarily large: 1) $u_{f} \rightarrow 1 / a_{0}$, where the $(+)$ pulse $\rightarrow \infty$ while the $(-)$ pulse remains fixed in magnitude; 2) $a_{0} \rightarrow \infty$, where both the $(+)$ and $(-)$ pulses become arbitrarily large in magnitude, but their maximum separation also decreases. 
Let us begin with the first possibility. We have that

$$
C_{13}\left(u, u^{\prime}\right)=\frac{\left(1-a_{0} u_{f}\right)^{4}}{8 \pi^{2}\left[u\left(1-a_{0} u_{f}\right)^{2}-u^{\prime}+a_{0} u_{f}^{2}\right]^{4}} \rightarrow 0
$$

as $u_{f} \rightarrow 1 / a_{0}$, with $u, u^{\prime}$, and $a_{0}$ fixed. Similarly,

$$
\begin{aligned}
C_{23}\left(u, u^{\prime}\right) & =\frac{\left(1-a_{0} u_{f}\right)^{4}}{8 \pi^{2}\left[u\left(1-a_{0} u_{f}\right)^{2}-\left(1-a_{0} u\right)\left(u^{\prime}-a_{0} u_{f}^{2}\right)\right]^{4}} \\
& \sim \frac{\left(1-a_{0} u_{f}\right)^{4}}{8 \pi^{2}\left[\left(1-a_{0} u\right)\left(u^{\prime}-a_{0} u_{f}^{2}\right)\right]^{4}} \rightarrow 0,
\end{aligned}
$$

as $u_{f} \rightarrow 1 / a_{0}$, if $u<u_{f}$, with $u, u^{\prime}, a_{0}$ fixed. In both of these cases, we find that the infinite $(+)$ pulse destroys correlations across the pulse.

Now we look at the $a_{0} \rightarrow \infty$ limit, where $u_{f} \rightarrow 0$. Therefore the only case of physical interest is $C_{13}\left(u, u^{\prime}\right)$. Let $u^{\prime}>u_{f}=\beta / a_{0}$, with $\beta<1$, and $u, u^{\prime}$ fixed. Then

$$
\begin{aligned}
C_{13}\left(u, u^{\prime}\right) & =\frac{(1-\beta)^{4}}{8 \pi^{2}\left[u(1-\beta)^{2}-u^{\prime}+\beta^{2} / a_{0}\right]^{4}} \\
& \sim \frac{(1-\beta)^{4}}{8 \pi^{2}\left[u(1-\beta)^{2}-u^{\prime}\right]^{4}},
\end{aligned}
$$

as $a_{0} \rightarrow \infty$. In this limit, the correlations do not vanish. Here one has large $(+)$ and $(-)$ mean pulses sitting nearly on top of one another, unlike in the $u_{f} \rightarrow 1 / a_{0}$ limit, where the pulse separation remains finite.

At this point we make some observations regarding $C_{12}\left(u, u_{f}\right)$. Let $u_{f}=1 / a_{0}-\epsilon$. Then

$$
C_{12}\left(u, u_{f}\right)=\frac{1}{8 \pi^{2}\left[\epsilon-\left(1 / a_{0}\right)+\epsilon a_{0} u\right]^{4}} \rightarrow \frac{a_{0}^{4}}{8 \pi^{2}},
$$

as $\epsilon \rightarrow 0$. Note that in this limit, where the final pulse is delayed as long as possible, $C_{12}$ becomes almost independent of $u$. Thus we seem to have the peculiar situation that $C_{12}\left(u, u_{f}\right)$ is almost independent of how far in the past $u$ is. However, we cannot strictly eliminate the $u$-dependence since we must always have $\epsilon>0$, in order to insure that the mirror's trajectory is always timelike. Were this not so, we might have the seemingly paradoxical situation in which two negative values of $u$, given by $\left(u_{1}, u_{2}\right)$, could be far apart so that $C_{11}\left(u_{1}, u_{2}\right)$ is small, but $C_{12}\left(u_{1}, u_{f}\right) \approx C_{12}\left(u_{2}, u_{f}\right) \approx a_{0}^{4} /\left(8 \pi^{2}\right)$.

\section{The Case $a_{0}<0$}

Here we will briefly discuss the case in which $a_{0}<0$, where the mirror accelerates to the left, emitting an initial $(+)$ energy pulse to the right, followed later by a final $(-)$ energy pulse. Our previous expressions for $C_{R R}$, Eqs. (38), (39), and (40), are still valid, but there is no longer any restriction on $u_{f}$. From Eq. (38), we can show that

$$
\frac{C_{12}\left(u, u^{\prime}\right)}{C_{\text {vac }}} \leq 0
$$


when $a_{0}<0$. Thus, again the effect of a $(+)$ energy pulse is to suppress correlations. Similarly, in this case, one finds

$$
\frac{C_{23}\left(u, u^{\prime}\right)}{C_{v a c}}>0
$$

so the correlations across the final (-) energy pulse are enhanced, just as they are for the leading $(-)$ energy pulse when $a_{0}>0$.

The behavior of $C_{13} / C_{v a c}$ is somewhat more complicated, and is essentially the inverse of the behavior for the $a_{0}>0$ case depicted in Fig. 3. That is, here $C_{13} / C_{v a c}$ starts at $u=u_{f}$ with a value less than one, increases monotonically through one, and reaches a limiting value of $\left(1-a_{0} u_{f}\right)^{4}>1$ as $u^{\prime} \rightarrow \infty$. This means that the correlation of a ray before the leading $(+)$ energy pulse and one long after the final $(-)$ energy pulse is enhanced, even though the $(+)$ energy pulse has the larger magnitude.

\section{Construction of the $C_{R L}$ 's}

Let us now consider the specific form of the $C_{R L}$ correlation functions for our piecewise trajectory. From Eq. (14), we have

$$
\begin{aligned}
C_{R L}\left(v, u^{\prime} \leq 0\right) & =\frac{1}{8 \pi^{2}\left(u^{\prime}-v\right)^{4}} \\
C_{R L}\left(v, 0 \leq u^{\prime} \leq u_{f}\right) & =\frac{1}{8 \pi^{2}\left[u^{\prime}-v\left(1-a_{0} u^{\prime}\right)\right]^{4}} \\
C_{R L}\left(v, u^{\prime} \geq u_{f}\right) & =\frac{\left(1-a_{0} u_{f}\right)^{4}}{8 \pi^{2}\left[u^{\prime}-a_{0} u_{f}^{2}-v\left(1-a_{0} u_{f}\right)^{2}\right]^{4}} .
\end{aligned}
$$

From Eq. (17) and the previous expressions, we obtain

$$
\begin{aligned}
& \frac{C_{R L}\left(v, u^{\prime}\right)}{C_{\text {static }}}=1 \quad\left(\text { for } u^{\prime} \leq 0\right), \\
& \frac{C_{R L}\left(v, u^{\prime}\right)}{C_{\text {static }}}=\frac{\left(u^{\prime}-v\right)^{4}}{\left[u^{\prime}-v\left(1-a_{0} u^{\prime}\right)\right]^{4}} \quad\left(\text { for } 0 \leq u^{\prime} \leq u_{f}\right), \\
& \frac{C_{R L}\left(v, u^{\prime}\right)}{C_{\text {static }}}=\frac{\left(1-a_{0} u_{f}\right)^{4}\left(u^{\prime}-v\right)^{4}}{\left[u^{\prime}-a_{0} u_{f}^{2}-v\left(1-a_{0} u_{f}\right)^{2}\right]^{4}}\left(\text { for } \quad u^{\prime} \geq u_{f}\right) .
\end{aligned}
$$

There are three regions for $v: v \leq 0$ - the ingoing $v$ ray arrives at the mirror before the acceleration phase begins; $0 \leq v \leq u_{f} /\left(1-a_{0} u_{f}\right)$ - the ingoing ray arrives during the constant

acceleration period; $v \geq u_{f} /\left(1-a_{0} u_{f}\right)$ - the ingoing ray arrives after the acceleration has ceased.

\section{E. Interpretation of the behavior of $C_{R L}$}

In this subsection, we will discuss how the behavior of $C_{R L}$ in various limits can be derived from kinematical considerations. We focus on the case $a_{0}>0$, with just brief remarks about the case $a_{0}<0$. From Eq. (56) , we see that for $0 \leq u^{\prime} \leq u_{f}<1 / a_{0}$ and for fixed $v<0$, 
with $a_{0}>0, C_{R L} / C_{\text {static }}$ increases as $u^{\prime}$ increases. Let us look at this further and focus on $C_{R L}\left(v, u^{\prime}\right)$ itself. We can rewrite Eq. (53) as

$$
C_{R L}\left(v, u^{\prime}\right)=\frac{1}{8 \pi^{2}\left[u^{\prime}\left(1+v a_{0}\right)-v\right]^{4}} .
$$

There are three possibilities: 1) $0>v>-a_{0}^{-1}$, so $1>1+v a_{0}>0$. Here $C_{R L}$ falls as $u^{\prime}$ increases, but more slowly than does $\left.C_{\text {static }}=1 /\left[8 \pi^{2}\left(u^{\prime}-v\right)^{4}\right] ; 2\right) v=-a_{0}^{-1}$, which implies that $C_{R L}=$ const independent of $u^{\prime}$; and 3) $v<-a_{0}^{-1}$. Here $C_{R L}$ grows as $u^{\prime}$ increases. We can understand all of these behaviors as arising from a competition between the Doppler blueshift factor $\left[p^{\prime}\left(u^{\prime}\right)\right]^{2}$, and the $1 /\left[p\left(u^{\prime}\right)-v\right]^{4}$ factor.

Recall our general expression, Eq. (14),

$$
C_{R L}\left(v, u^{\prime}\right)=\frac{\left[p^{\prime}\left(u^{\prime}\right)\right]^{2}}{8 \pi^{2}\left[p\left(u^{\prime}\right)-v\right]^{4}} .
$$

As $u^{\prime}$ increases, here $\left[p^{\prime}\left(u^{\prime}\right)\right]^{2}$ increases because the mirror is accelerating to the right. At the same time, $p\left(u^{\prime}\right)$ is a monotonic increasing function of $u^{\prime}$, so $1 /\left[p\left(u^{\prime}\right)-v\right]^{4}=1 /\left[p\left(u^{\prime}\right)+|v|\right]^{4}$ decreases as $u^{\prime}$ increases. This decrease simply reflects the fact that the incoming fluctuations are more weakly correlated as $p\left(u^{\prime}\right)-v$ increases. The two effects exactly balance when $v=-a_{0}^{-1}$, as seen from Eq. (58). We can understand why for more negative $v$, the effect of the $1 /\left[p\left(u^{\prime}\right)-v\right]^{4}$ term is less important, as follows. The more negative $v$ is (larger $|v|$ is), the smaller this factor will be for a given value of $p\left(u^{\prime}\right)$. However, a given change in $p\left(u^{\prime}\right)$ will cause $1 /\left[p\left(u^{\prime}\right)-v\right]^{4}$ to change by a smaller fraction when $|v|$ is larger. (Conversely, when $|v|$ is small, a small change in $p\left(u^{\prime}\right)$ produces a large fractional change in $1 /\left[p\left(u^{\prime}\right)-v\right]^{4}$.)

Now let us turn to the peak value of $C_{R L} / C_{\text {static }}$ at $u^{\prime}=u_{f}$ for various values of $v<0$. The value at the peak is

$$
\frac{C_{R L}\left(v, u_{f}\right)}{C_{\text {static }}\left(v, u_{f}\right)}=\frac{\left[p^{\prime}\left(u_{f}\right)\right]^{2}\left(u_{f}-v\right)^{4}}{\left[p\left(u_{f}\right)-v\right]^{4}} .
$$

The factor $\left[p^{\prime}\left(u_{f}\right)\right]^{2}=\left(1-a_{0} u_{f}\right)^{-4}$ is independent of $v$, so we can ignore it here. From Eq. (28) we have that

$$
p\left(u_{f}\right)=\frac{u_{f}}{1-a_{0} u_{f}}>u_{f} .
$$

Therefore we need to look at

$$
\frac{\left(u_{f}-v\right)^{4}}{\left[p\left(u_{f}\right)-v\right]^{4}}=\frac{\left(|v|+u_{f}\right)^{4}}{\left[|v|+p\left(u_{f}\right)\right]^{4}} .
$$

As $|v|$ increases, this ratio varies smoothly from $u_{f}^{4} /\left[p\left(u_{f}\right)\right]^{4}<1$ to 1 . This explains the increase in the peak $\left(u^{\prime}=u_{f}\right)$ value, which eventually saturates at a value of $\left[p^{\prime}\left(u_{f}\right)\right]^{2}$, which increases as $u_{f}$ increases for fixed $a_{0}$.

Note that in the special case of $v=0$, over the interval $0<u^{\prime}<u_{f}, C_{R L} / C_{\text {static }}=$ const $=1$. This follows from Eq. (56). In this case there is no peak, since $C_{R L} / C_{\text {static }}$ falls monotonically for $u^{\prime}>u_{f}$.

Let us comment briefly on the case $a_{0}<0$. Here again there is a competition between the $p\left(u^{\prime}\right)$ and the $p\left(u^{\prime}\right)-v$ factors, but each is in the opposite direction compared to when $a_{0}>0$. Thus $p\left(u^{\prime}\right)$ is now a redshift factor, but the incoming rays are more strongly correlated as 


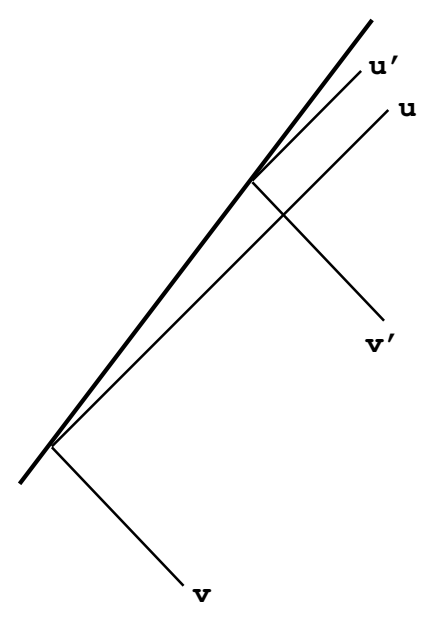

FIG. 4: The reflection of null rays by a mirror moving at a constant velocity to the right.

$p\left(u^{\prime}\right)-v$ decreases. The net effect in a typical case is to cause $C_{R L} / C_{\text {static }}$ to fall to a minimum value, and then to rise again, the opposite behavior as when $a_{0}>0$.

Now let us return to the case $a_{0}>0$ and where one ray arrives at the mirror before the acceleration $(v=u<0)$ and one arrives there afterwards, $u^{\prime}>u_{f}$. Note that here $C_{13}\left(u, u^{\prime}\right) \leftrightarrow C_{R L}\left(v, u^{\prime}\right)$, when $u \leftrightarrow v$, because $p^{\prime}(u)=1$ for $v \leq 0$. From Eq. (40), we had

$$
\frac{C_{13}\left(u, u^{\prime}\right)}{C_{v a c}}=\frac{\left(1-a_{0} u_{f}\right)^{4}\left(u-u^{\prime}\right)^{4}}{\left[u\left(1-a_{0} u_{f}\right)^{2}-u^{\prime}+a_{0} u_{f}^{2}\right]^{4}} .
$$

This is larger than 1 for $u^{\prime}=u_{f}$, and then drops below 1 at $u^{\prime}=u_{c}^{\prime}=u_{f}-u\left(1-a_{0} u_{f}\right)$.

Consider the following two limits:

$$
\frac{C_{13}\left(u, u^{\prime}\right)}{C_{\text {vac }}} \sim\left\{\begin{array}{cc}
1 /\left(1-a_{0} u_{f}\right)^{4}>1, & u \rightarrow-\infty, u^{\prime} \text { fixed } \\
\left(1-a_{0} u_{f}\right)^{4}<1, & u^{\prime} \rightarrow+\infty, u \text { fixed }
\end{array} .\right.
$$

Thus for $|u|$ large, $C_{13} / C_{v a c}$ starts above 1 and eventually falls to a constant value $<1$ as $u^{\prime}$ grows, as illustrated in Fig. 3. To understand these limits, we use the correspondence between $C_{13}\left(u, u^{\prime}\right)$ and $C_{R L}\left(v, u^{\prime}\right)$, and consider a mirror which always moves to the right at a constant velocity equal to the final velocity of the accelerated mirror. Then we have

$$
p\left(u^{\prime}\right)=\frac{u^{\prime}}{\left(1-a_{0} u_{f}\right)^{2}}+\text { const }
$$

and

$$
C_{R L}\left(v, u^{\prime}\right)=\frac{\left(1-a_{0} u_{f}\right)^{-4}}{8 \pi^{2}\left[u^{\prime}\left(1-a_{0} u_{f}\right)^{-2}-v\right]^{4}} \propto \frac{\left(1-a_{0} u_{f}\right)^{4}}{u^{\prime 4}}, \text { as } u^{\prime} \rightarrow \infty .
$$

There are two competing effects here: the $\left(1-a_{0} u_{f}\right)^{-4}>1$ factor in the numerator is a blueshift factor. However, it is dominated by the $\left[\left(1-a_{0} u_{f}\right)^{-2}\right]^{4}$ factor in the denominator. The faster the mirror is moving, the larger $v^{\prime}-v$ is for a given $u^{\prime}-u$, as illustrated in Fig. 4, This tends to cause the correlation function between, e. g., $v$ and $u^{\prime}$, to be smaller because it is proportional to $1 /\left(v^{\prime}-v\right)^{4}$. This is the origin of the $1 /\left[p\left(u^{\prime}\right)-v\right]^{4}$ factor in $C_{R L}$ that 


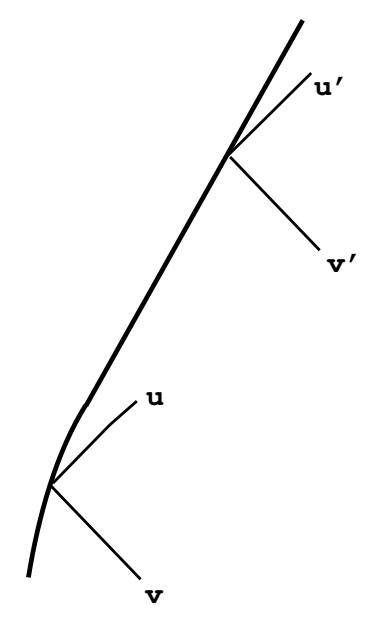

FIG. 5: Here the left-moving ray $v$ reflects from the mirror while it is still accelerating, while the ray $v$ reflects from it long after the acceleration has ceased.

dominates the $\left[p^{\prime}\left(u^{\prime}\right)\right]^{2}$ factor for large $u^{\prime}$. Another way to say this is: as we consider a sequence of mirrors, each moving faster to the right, a fixed $u^{\prime}, v^{\prime}$ corresponds to a larger difference $v^{\prime}-v$ and hence a weaker correlation.

Let us now return to our piecewise accelerating trajectory. For the second limit in Eq. (64), as $u^{\prime} \rightarrow \infty$ for fixed $u$, the trajectory becomes very close to that of a mirror which is always inertial at the final velocity (see Fig. (5)), and gives $C_{13} / C_{\text {vac }}=C_{R L} / C_{\text {static }}=$ $\left(1-a_{0} u_{f}\right)^{4}$. In this case the Doppler blueshift at $u^{\prime}$ is dominated by the stretching of the difference of the $v$ values of incoming rays. Similarly, the fall in the ratio after acceleration stops reflects the fact that it must asymptotically approach the value for an eternally inertial mirror at the final velocity. This value is smaller for faster mirrors.

For the first limit in Eq. (64), as $u \rightarrow-\infty$ for $u^{\prime}$ fixed, the trajectory becomes close to that of an eternally static mirror (see Fig. [6), except for the blueshift factor at $u^{\prime}$. The difference $v^{\prime}-v$ for this trajectory is close to that for the eternally static mirror. However, there is still a $\left(1-a_{0} u_{f}\right)^{-4}$ factor at $u^{\prime}$, which produces the result for this limit.

Which regime we are in, i.e., the first limit or the second, is determined by the relative magnitudes of $u\left(1-a_{0} u_{f}\right)^{2}$ and $u^{\prime}$ in Eq. (63) . When $\left|u\left(1-a_{0} u_{f}\right)^{2}\right| \gg u^{\prime}$, we are in the large $|u|$ limit. Eventually $u^{\prime}$ grows so that $\left|u\left(1-a_{0} u_{f}\right)^{2}\right| \ll u^{\prime}$, and we are in the other limit. However, the larger $|u|$ is, the longer it takes to go from one regime to the other. This explains why $u_{c}^{\prime}$ grows as $|u|$ grows. Recall that this was a question which we posed in Sec. IIIC] as to why the decay depicted in Fig. [3 is slower for earlier initial rays.

\section{SUMMARY AND DISCUSSION}

In this paper, we have studied the flux correlation functions $C_{R R}\left(u, u^{\prime}\right)$ and $C_{R L}\left(v, u^{\prime}\right)$ for the mirror trajectory illustrated in Fig. 2. In all cases, these correlation functions describe how the initial vacuum fluctuations are processed by the mirror. Flux fluctuations reflect from the mirror and undergo a redshift or blueshift. At the same time, the temporal separation between a pair of rays can be increased or decreased by reflection. Let us recall the main results for $C_{R R}\left(u, u^{\prime}\right)$, the correlation function between a pair of right-moving fluxes which 


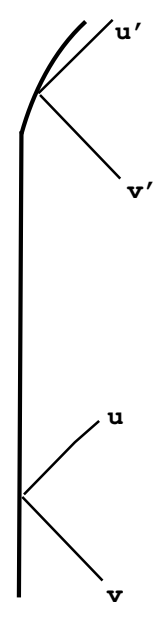

FIG. 6: Here the left-moving ray $v$ reflects from a mirror at rest. This mirror subsequently begins to accelerate, and then the left-moving ray $v^{\prime}$ reflects from it.

have both reflected from the mirror. This function differs from that in the vacuum state only if $u$ and $u^{\prime}$ are separated by one or both delta-function pulses in the mean stress tensor. If they are separated by only the $(-)$ energy pulse, the correlations are enhanced, whereas if they are separated by only the $(+)$ energy pulse, the correlations are suppressed compared to those in the vacuum. If both pulses lie between $u$ and $u^{\prime}$, then the correlations can be either suppressed or enhanced, depending on the proximity of $u^{\prime}$ to the second pulse. In the limit that the $(+)$ energy pulse becomes very large (by letting $u_{f} \rightarrow 1 / a_{0}$ ), the correlations across this pulse are destroyed and $C_{R R}\left(u, u^{\prime}\right) \rightarrow 0$.

In the special case where $u$ and $u^{\prime}$ lie on the mean pulses, one finds that the correlation function takes the vacuum value, $C_{R R}\left(0, u_{f}\right)=C_{v a c}\left(0, u_{f}\right)$. This means that the flux fluctuations around these pulses are no more and no less correlated than are flux fluctuations in the vacuum state. This may come as a surprise in light of the discussion in Sect. I to the effect that the time integrated flux can never fluctuate below zero. However, there are two points worth noting. The first is that flux fluctuations in the vacuum must guarantee that the integrated flux in any measurement is not only non-negative, but in fact zero. Thus the degree of correlation in the vacuum state, which suffices to guarantee a vanishing integrated energy in the vacuum state, also suffices to guarantee non-negative integrated energy flux for a moving mirror. The second point is that if the negative pulse fluctuates below its mean, the compensating positive fluctuation does not have to occur near the mean positive pulse, but could be elsewhere.

One of the lessons of this study is that there is great deal more happening in the accelerating mirror geometry than is revealed by the expectation value of the stress tensor. There are subtle increases or reductions in correlations between the flux along rays where the expectation value vanishes.

In this paper, we have restricted our attention to the study of the correlations of distinct regions. More generally, one would like to study the correlation of measurements made in overlapping regions. This requires integration of the correlation function through the singularity at coincident points. Integration by parts can be employed to define the otherwise divergent integrals [26, 27]. This will be treated in the context of moving mirror spacetimes in a future work. 


\section{Acknowledgments}

We would like to thank Chris Fewster for helpful discussions. This work was supported in part by the National Science Foundation under Grants PHY-0244898 and PHY-0139969.

\section{APPENDIX A}

In this appendix, we will show that uniform acceleration is the most general trajectory for which the $C_{R R}\left(u, u^{\prime}\right)$ correlation function has the same form as for the vacuum state. We want to ask when

$$
C_{R R}\left(u, u^{\prime}\right)=\frac{\left[p^{\prime}(u) p^{\prime}\left(u^{\prime}\right)\right]^{2}}{8 \pi^{2}\left[p(u)-p\left(u^{\prime}\right)\right]^{4}}=C_{v a c}\left(u, u^{\prime}\right)=\frac{1}{8 \pi^{2}\left(u-u^{\prime}\right)^{4}},
$$

which implies that

$$
\frac{\left[p^{\prime}(u) p^{\prime}\left(u^{\prime}\right)\right]}{\left[p(u)-p\left(u^{\prime}\right)\right]^{2}}=\frac{1}{\left(u-u^{\prime}\right)^{2}} .
$$

Note that we take the $(+)$ sign when taking the square root; this is correct because $p(u)$ is a monotonic increasing function, so $p^{\prime}(u)>0$ and $p^{\prime}\left(u^{\prime}\right)>0$.

If we write Eq. (A2) as

$$
-\frac{d}{d u}\left[\frac{p^{\prime}\left(u^{\prime}\right)}{p(u)-p\left(u^{\prime}\right)}\right]=\frac{1}{\left(u-u^{\prime}\right)^{2}}
$$

and integrate, we get

$$
p(u)=p^{\prime}\left(u^{\prime}\right) \frac{\left(u-u^{\prime}\right)}{\left[1-c_{1}\left(u^{\prime}\right)\left(u-u^{\prime}\right)\right]}+p\left(u^{\prime}\right),
$$

where $c_{1}\left(u^{\prime}\right)$ is a function of $u^{\prime}$ alone.

From this expression, we see that $p(u)$ for fixed $u^{\prime}$ must be the ratio of two linear polynomials of $u$. Let

$$
\begin{aligned}
p(u) & =\frac{u+b_{0}}{b_{1} u+b_{2}} \\
p^{\prime}(u) & =\frac{b_{2}-b_{1} b_{0}}{\left(b_{1} u+b_{2}\right)^{2}} .
\end{aligned}
$$

and similarly

$$
p\left(u^{\prime}\right)=\frac{u^{\prime}+b_{0}}{b_{1} u^{\prime}+b_{2}},
$$

where $b_{0}, b_{1}$, and $b_{2}$ are constants. If these relations are put back into Eq. (A2), we find that it is satisfied for all choices of $b_{0}, b_{1}$, and $b_{2}$. Thus the above $p(u)$ is the most general form for which $C_{R R}\left(u, u^{\prime}\right)=C_{v a c}\left(u, u^{\prime}\right)$. From Eq. (21), which can be rewritten to the form of Eq. (A7), this is just the case of uniform acceleration. The three constants $b_{0}, b_{1}, b_{2}$ are related to the magnitude of the acceleration, the time at which the mirror starts to accelerate, and its position when it starts.

[1] L.H. Ford, Proc. Roy. Soc. Lond. A 364, 227 (1978). 
[2] L.H. Ford, Phys. Rev. D 43, 3972 (1991).

[3] L.H. Ford and T.A. Roman, Phys. Rev. D 51, 4277 (1995), gr-qc/9410043.

[4] L.H. Ford and T.A. Roman, Phys. Rev. D 55, 2082 (1997), gr-qc/9607003.

[5] E.E. Flanagan, Phys. Rev. D, 56, 4922 (1997), gr-qc/9706006.

[6] M.J. Pfenning and L.H. Ford, Phys. Rev. D 55, 4813 (1997), gr-qc/9608005.

[7] M.J. Pfenning and L.H. Ford, Phys. Rev. D 57, 3489 (1998), gr-qc/9710055.

[8] C.J. Fewster and S.P. Eveson, Phys. Rev. D 58, 084010 (1998), gr-qc/9805024.

[9] C.J. Fewster, Class. Quantum Grav. 17, 1897 (2000), gr-qc/9910060.

[10] S.A. Fulling and P.C.W. Davies, Proc. R. Soc. London A348, 393 (1976).

[11] P.C.W. Davies and S.A. Fulling, ibid, A356, 237 (1977).

[12] L.H. Ford and T.A. Roman, Phys. Rev. D 60, 104018 (1999), gr-qc/9901074.

[13] R. Carlitz and R. Willey, Phys. Rev. D 36, 2327 (1987).

[14] N. Obadia and R. Parentani, Phys. Rev. D 64, 044019 (2001), gr-qc/0103061.

[15] N. Obadia and R. Parentani, Phys. Rev. D 67, 024021 (2003), gr-qc/0208019.

[16] N. Obadia and R. Parentani, Phys. Rev. D 67, 024022 (2003), gr-qc/0209057.

[17] L.H. Ford, Ann. Phys (NY) 144, 238 (1982).

[18] Chung-I Kuo and L.H. Ford, Phys. Rev. D 47, 4510 (1993), gr-qc/9304008.

[19] E. Calzetta and B.L. Hu, Phys. Rev. D 49, 6636 (1993), gr-qc/9312036; Phys. Rev. D 52, 6770 (1995), gr-qc/9505046.

[20] E. Calzetta, A. Campos, and E. Verdaguer, Phys. Rev. D 56, 2163 (1997), gr-qc/9704010.

[21] N.G. Phillips and B.L. Hu, Phys. Rev. D 55, 6123 (1997), gr-qc/9611012.

[22] R. Martin and E. Verdaguer, Phys. Rev. D 60, 084008 (1999), gr-qc/9904021.

[23] C.-H. Wu and L.H. Ford, Phys. Rev. D 60, 104013 (1999), gr-qc/9905012

[24] C. Barrabes, V. Frolov, and R. Parentani, Phys. Rev. D 62, 044020 (2000), gr-qc/0001102.

[25] N.G. Phillips and B.L. Hu, Int. J. Theor. Phys. 39, 1817 (2000), gr-qc/0004006 Phys.Rev. D 62,084017 (2000), gr-qc/0005133.

[26] C.-H. Wu and L. H. Ford, Phys. Rev. D 64, 045010 (2001), quant-ph/0012144.

[27] L. H. Ford and C.-H. Wu, Int. J. Theor. Phys. 42, 15 (2003), gr-qc/0102063.

[28] An individual measurement of the time-integrated energy might be made, for example, by a detector switched on in the far past and switched off in the far future.

[29] N.D. Birrell and P.C.W. Davies, Quantum Fields in Curved Space, Cambridge University 
Press, 1982, p. 105. 